\title{
Bacterial Communities in the Water of Larval-Rearing System for the Yesso Scallop (Patinopecten Yessoensis)
}

\author{
Ma $\mathrm{Y}^{1 *}$, Liang J ${ }^{2}$, Zhao $\mathrm{X}^{2}$, Li $\mathrm{M}^{2}$, Sun $\mathrm{X}^{1}$ and Liu J ${ }^{1}$ \\ ${ }^{1}$ Key Laboratory of Mariculture \& Stock Enhancement in North China's Sea of \\ Ministry of Agriculture, Dalian Ocean University, Dalian 116023, China \\ 2Zhangzi island Group Company Limited, Dalian 116001, China
}

\section{Review Article}

Volume 1 Issue 2

Received Date: April 03, 2017

Published Date: April 19, 2017

*Corresponding author: Yuexin Ma, Key Laboratory of Mariculture \& Stock Enhancement in North China's Sea of Ministry of Agriculture, Dalian Ocean University, Dalian 116023, China, Tel: +86-41184763096; E-mail: mayuexin_1963@163.com

\section{Abstract}

Bacterial disease is a significant issue for larviculture of the Yesso scallop (Patinopecten yessoensis). One source of bacteria is the water of larval-rearing tanks. In this study, water bacterial communities were investigated by MiSeq sequencing during larval development at fertilized egg W1, trochophora W2, D-shaped larvae W3, umbo larvae W4, juvenile scallop W5 stages. Genomic DNA was extracted from the water samples, and a gene segment from the V3-V4 portion of the 16S rRNA gene was amplified and sequenced using an Illumina Miseq sequencer. A total of 69245 optimized reads obtained from five samples were identified as 9 phyla and 86 genera. Bacterial communities were dominated by the Proteobacteria and Bacteroidetes at phylum level across five larval development stages. At genus level, sequences assigned to Pseudoalteromonas, NS3a marine group and Sulfitobacter were dominant at stages of W1, W4 and W5. Moreover, reads from Vibrio became dominant at stage W2, followed by Pseudoalteromonas and Sulfitobacter; Glaciecola was dominant at stage W3, followed by NS3a marine group and Sulfitobacter. These findings indicated that water bacterial communities changed greatly during the trochophora and D-shaped larvae stages of larval development.

Keywords: Patinopecten yessoensis; Bacterial community; 16S rRNA; High-throughput sequencing

\section{Introduction}

The Yesso scallop Patinopecten yessoensis has become one of the major cultured species in Liaoning and Shandong Provinces, China, making an important contribution to the socioeconomic development of these coastal regions. The larval stage of scallop is susceptible to mass mortality with opportunistic pathogens [1]. The successful production of healthy scallop larvae, to a large extent, depends on the quality of water including the species of bacteria present. Sandaa et al. studied the bacterial communities in the Great scallop Pecten maximus aquaculture systems using denaturing gradient gel electrophoresis (DGGE) [2]. As indicated by DGGE, 


\section{International Journal of Oceanography \& Aquaculture}

there are high similarities in the bacterial community compositions among different larvae aquaculture systems (stagnant, flow-through and a flow- through system with seawater treated with ozone) [2]. The dominant culturable genera in the water of Chilean scallop Argopecten purpuratus larval rearing tanks and the survival of larvae are different in the presence and absence of antibiotic [3].

Massively parallel next generation sequencing platforms have now been successfully utilized to characterize seawater bacterial communities [4-6]. Sun et al. (2016) compared the bacterial communities associated with five Yesso scallop larval development stages by Illumina high-throughput sequencing, and found that the larval mortality occurred during the transition from the D-shaped to umbo larvae and across the entire period of umbo larvae stage coincided with the great change of bacterial community [7]. Vibrio splendidus and Vibrio tasmaniensis are proved to be the pathogens causing mortality in Yesso scallop larvae [1]. However, much less is known about the bacterial composition in the water of Yesso scallop larval-rearing tanks. It is also unclear that whether the bacteria in the water are related to the larval mortality. The aim of this study was to characterize and compare water bacterial communities at different larval development stages of the Yesso scallop using Illumina high-throughput sequencing, providing the basis for the management of the scallop seedling system.

\section{Materials and Methods}

\section{Sample Collection and Processing}

Water samples were collected from the Yesso scallop hatchery in Zhang Zidao Island Group Co. Ltd., Dlian, China between March and April 2014. After filtration and precipitation, seawater was used in the hatchery. Larvae were cultured in plastic tanks containing $100 \mathrm{~L}$ seawater under static conditions at a density of 5-6 larvae/mL and fed Dicrateria inornata $100 \mathrm{~mL}, 7-9 \times 10^{5}$ cells $/ \mathrm{mL}$ three times a day. Low-pressure electrical blowers provided aeration via air stones. Water temperature was kept at 15 $\pm 1^{\circ} \mathrm{C}$ and salinity at $32 \pm 0.5$ PSU (Practial Salinity Units). Half $(50 \%)$ of the seawater each tank was replaced every day. During larval development stages, three water samples of approximately $500 \mathrm{~mL}$ each tank were pooled and filtered onto $0.22-\mu \mathrm{m}$ filters which were stored frozen at $-80^{\circ} \mathrm{C}$ until required for DNA extraction.

\section{DNA Extraction and PCR Amplification}

Genomic DNA was extracted using a soil DNA extraction kit (Shanghai Sangon Biological Engineering Technology and Services Company Limited, Shanghai,
China) A gene segment (V3-V4 portion) of 16S rRNA gene was amplified through PCR by pre-denaturing at $95^{\circ} \mathrm{C}$ for 2 min followed by 30 cycles of denaturing at $95^{\circ} \mathrm{C}$ for $30 \mathrm{~s}$, annealing at $55^{\circ} \mathrm{C}$ for $30 \mathrm{~s}$ and extending at $72^{\circ} \mathrm{C}$ for $45 \mathrm{~s}$, and an additional extension cycle at $72^{\circ} \mathrm{C}$ for $10 \mathrm{~min}$. The gene was amplified with primers $338 \mathrm{~F}$ (5'-barcodeACTCCTACGGGAGGCAGCA-3') and 806R (5'GGACTACHVGGGTWTCTAAT-3'), in which barcode is an eight-base sequence unique to each sample [8-9]. PCR was performed in triplicate and the reaction volume was $20 \mu \mathrm{L}$ containing $4 \mu \mathrm{L}$ of $5 \times$ Fast Pfu buffer, $2 \mu \mathrm{L}$ of $2.5 \mathrm{mM}$ dNTP (each nucleotide), $0.4 \mu \mathrm{L}$ of $5 \mu \mathrm{M}$ primers (each direction), $0.4 \mu \mathrm{L}$ of Fast Pfu polymerase $(2.5 \mathrm{U} / \mu \mathrm{L})$, and $10 \mathrm{ng}$ of template DNA.

\section{Illumina MiSeq Sequencing}

Amplicons were extracted from 2\% agarose gels and purified using the Axyprep DNA Gel Extraction Kit (Axygen Biosciences, Union City, CA, U.S.) according to the manufacturer's instructions and quantified using QuantiFluor $^{\mathrm{TM}}$-ST (Promega, U.S.). Purified amplicons were pooled in equimolar and paired-end sequenced $(2 \times$ 300 ) on an Illumina MiSeq platform following the standard protocols (Majorbio Bio-Pharm Technology Co., Ltd., Shanghai, China).

\section{Processing of Sequencing Data}

Raw fastq files were demultiplex and quality-filtered using QIIME (version 1.17) [10] with the following criteria: (i) $300 \mathrm{bp}$ reads truncated at any site over a 50 bp sliding window were scored in average $<20$, truncated reads shorter than $50 \mathrm{bp}$; (ii) any mismatched in barcode region, 2 nucleotide mismatched in primer and reads containing ambiguous characters (iii) only sequences that overlap longer than $10 \mathrm{bp}$ assembled, and reads failed to be assembled discarded. Operational Taxonomy Units (OTUs) were clustered with $97 \%$ similarity cutoff using UPARSE (version $7.1 \mathrm{http} / / /$ drive5.com/uparse/) [11] and chimeric sequences were identified and removed using UCHIME [12]. The most abundant sequence each OTU was chosen as the representative. The taxonomy of each 16S rRNA gene sequence was analyzed by RDP Classifier (http://rdp.cme.msu.edu/) [13] against the silva (SSU115)16S rRNA database [14] using confidence threshold of 70\% [15]. Rarefaction analysis, Coverage (the Good's coverage), Ace (the ACE estimator), Chao (the Chao1 estimator), Shannon and Simpson indices were created using mothur software (version v.1.30.1 http://www.mothur.org/wiki/Schloss_SOP\#Alpha_divers ity). Venn, mcrobial community barplot, heatmap and principal component analysis (PCA) were performed in R software. 


\section{International Journal of Oceanography \& Aquaculture}

\section{Results}

\section{Illumina Miseq Sequencing Data}

A total of 69245 optimized reads were obtained from water samples .Rarefaction curves generated at the OTUs level were approached saturation, indicating that the sequencing depth was enough to reflect the diversity. These reads were clustered into 209 OTUs and the number of OTUs of each water sample ranged from 166 to 188 (Table 1). Good's coverage estimations revealed that $99.76 \%$ to $99.84 \%$ of the species were retrieved (Table 1 ).
Our analysis revealed 9 phyla, 17 classes, 33 orders, 50 families and 86 genera in total.

\section{Species Richness and Diversity Estimates}

Bacterial taxonomic richness and diversity were different among five water samples. As indicated by Chao and Ace indices (Table 1), W3 stage harbored the highest community richness, followed by W2, W4 and W1 stages, while the lowest community richness was found at W5 stage. Shannon and Simpson indices (Table 1) showed that the bacterial diversity increased in order of stages $\mathrm{W} 1, \mathrm{~W} 4, \mathrm{~W} 3, \mathrm{~W} 5$ and $\mathrm{W} 2$.

\begin{tabular}{|c|c|c|c|c|c|c|}
\hline Sample & OTUs & Coverage (\%) & Ace & Chao & Shannon & Simpson \\
\hline W1 & 170 & 99.76 & 189 & 194 & 2.67 & 0.19 \\
\hline W2 & 179 & 99.8 & 199 & 196 & 3.26 & 0.08 \\
\hline W3 & 188 & 99.84 & 210 & 208 & 2.94 & 0.12 \\
\hline W4 & 179 & 99.82 & 194 & 194 & 2.8 & 0.17 \\
\hline W5 & 166 & 99.81 & 176 & 173 & 3.13 & 0.11 \\
\hline
\end{tabular}

Table 1: Alpha diversity obtained from five water samples.

\section{Community Composition}

The shared OTUs were shown via Venn diagram (Figure 1). Each stage contained only a minority of special OTUs (0 3). Most of OTUs (147 167) were shared between stages

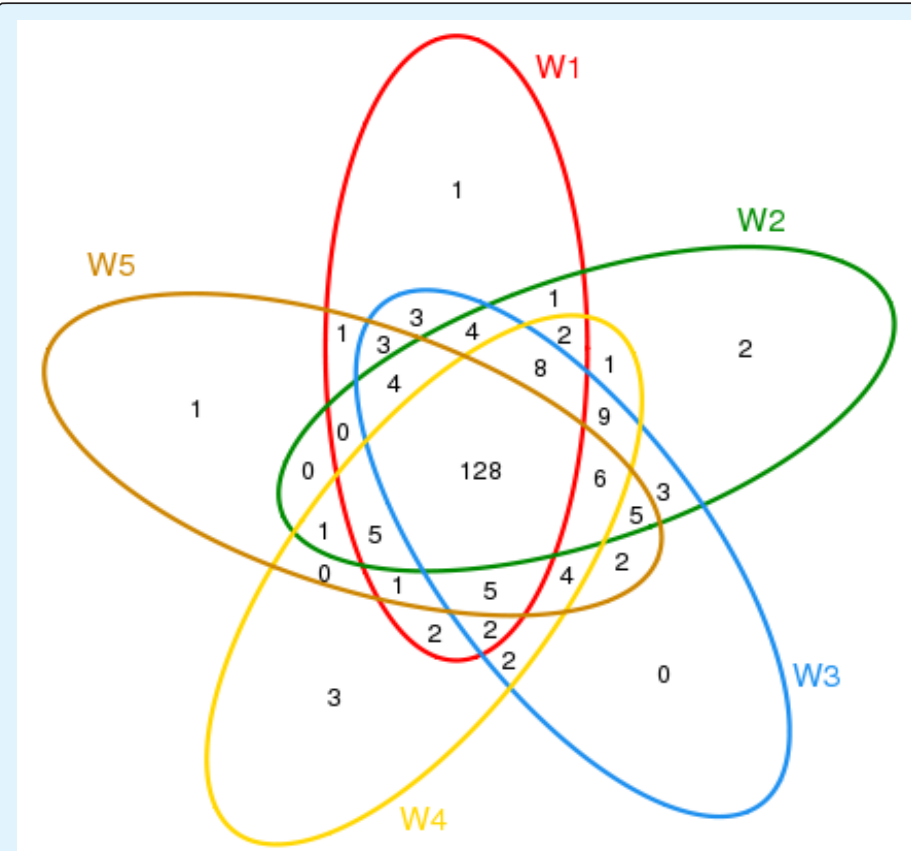

Figure 1: Venn diagram showing OTUs unique to and shared by Yesso scallop larval development stages.
Nine identified phyla (Proteobacteria, Bacteroidetes, Cyanobacteria, Actinobacteria, Planctomycetes, Chlamydiae, Verrucomicrobia, Firmicutes and Fusobacteria) and two candidate divisions (BD1-5 and OD1) were detected among 5 stages, with the bacterial 16S rDNA sequences dominated by Proteobacteria $(74.87 \% \sim 84.53 \%)$ and Bacteroidetes $(14.49 \% \sim$ $24.43 \%)$. The other 7 phyla and candidate division OD1were represented by less than $1 \%$ of the total sequence reads. Sequences from candidate division BD1-5 and phyla Cyanobacteria, Actinobacteria, Planctomycetes, Chlamydiae and Verrucomicrobia were found at all stages. Sequences representing candidate division OD1 existed at W1, W2 and W4 stages. Sequences from Fusobacteria were only detected at W4 stage and those from Firmicutes were found at W5 stage alone.

Analyzing at genus level revealed 86 different identified genera in total. Sequence proportions of 12 genera, NS3a marine group, Roseobacter clade DC-80-3 lineage, Roseobacter clade AS-21 lineage, SAR11 Surface 1 and BD1-5 were greater than 1\% (Figure 2). Apparent shift in Pseudoalteromonas, Glaciecola and Vibrio was observed across different larval development stages (Figure 2).

Sequences assigned to Pseudo alteromonas (40.07\%, $37.88 \%, 25.96 \%)$, NS3a marine group (16.62\%, $11.27 \%$, $17.47 \%)$ and Sulfitobacter $(8.99 \%, 8.89 \%, 8.21 \%)$ were dominant at stages of W1, W4 and W5; followed by those 
to Alteromonas (3.40\%) and Vibrio (2.91\%) at stage W1; Alteromonas (6.73\%) and Phaeobacter (4.72\%) at stage W4 and Roseobacter clade DC-80-3 lineage (6.20\%) and Alteromonas $(4.55 \%)$ at stage W5. However, reads from Vibrio (19.33\%) became dominant at stage W2, followed by those to Pseudoalteromonas(15.31\%), Sulfitobacter (10.26\%), NS3a marine group (8.78\%), Aliivibrio (6.10\%) and Roseobacter clade DC-80-3 lineage (5.40\%); while sequences belonged to Glaciecola (27.90\%) was dominant at stage W3, followed by those to NS3a marine group (15.40\%), Sulfitobacter (7.87\%), Polaribacter (5.43\%), Alteromonas (5.38\%) and Roseobacter clade DC-80-3 lineage $(5.32 \%)$.

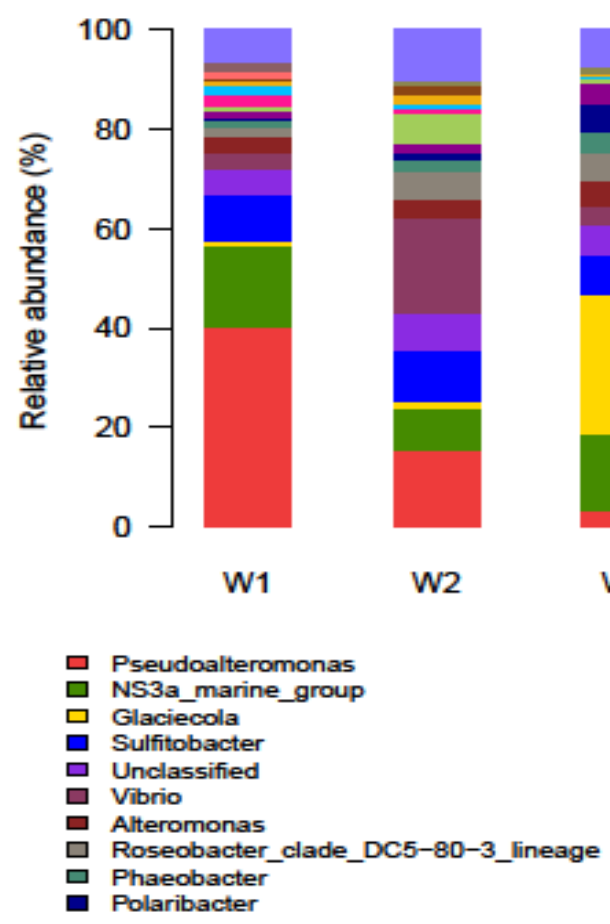



W3


W4

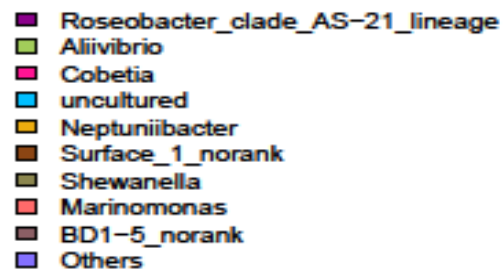

Figure 2: Genus abundance (\%) of water at Yesso scallop larval development stages.

Note: sequences abundance less than $1 \%$ at a stage was combined into "others".

\section{PCA of Phylogenetic Relatedness}

PCA score plot showed that the first principal component (PC1), which explained $74.92 \%$ of the variance in the bacterial communities, separated the W3 stage and other stages (Figure 3). The W4 stage was closely related to W1 stage, whereas the W2 stage was separate from the other stages along PC2, which represented $20.36 \%$ of the total variations (Figure 3 ).

Overall, the PC1 and PC2 axes explained $95.28 \%$ of the variations between the different bacterial communities. Hierarchically clustered heatmap analysis based on the bacterial community profiles at genus level disclosed that W1 and W4 stages grouped together firstly, and they then clustered with W5, W2 and W3 stages in order (Figure 4).

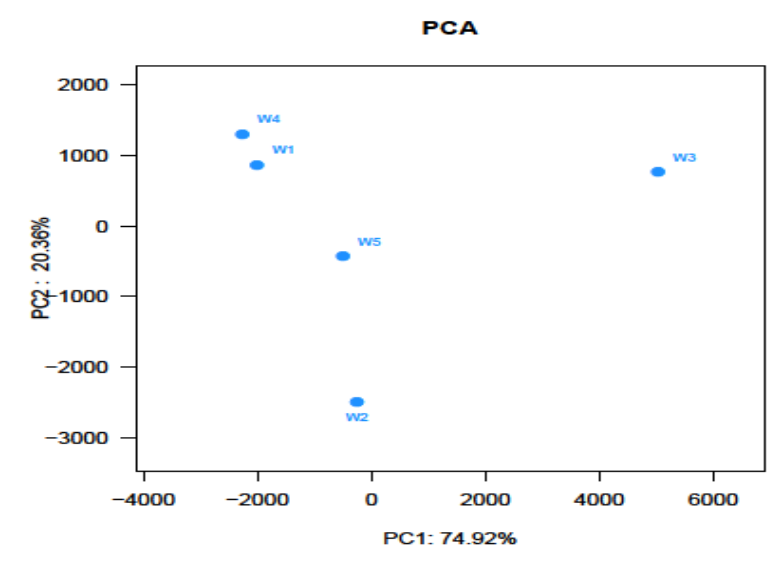

Figure 3: Principal component analysis of bacterial communities among five stages at OTU level. 




Figure 4: Heatmap based on the genus abundance similarity.

Note: Double hierarchical dendrogram shows the bacterial distribution among 5 stages. 


\section{International Journal of Oceanography \& Aquaculture}

The heatmap plot depicts the relative percentage each genus (rows) at each stage (columns). The relative abundance of bacterial genera is depicted by color intensity with the legend appeared at the bottom of the figure. Clusters based on the distance of 5 stages along Xaxis and the bacterial genera along $\mathrm{Y}$-axis are indicated in the upper and left of the figure, respectively.

\section{Discussion}

During different development stages of Yesso scallop larvae, the bacterial communities in the water of larvalrearing tanks were diverse, especially, at stages W3 and W2 (Figure 2-4). One of the reasons may be the change of the bacterial compositions from inlet water $[2,16]$. Similarly, the communities in treated clean seawater used in the oyster (Crassostrea gigas) hatchery are variable on scales of days [16]. Nevertheless, only minor variations in the bacterial community composition in DGGE profile have been found in the water collected at different days during the growth of the Great scallop larvae [2].

The bacterial communities observed in water samples collected in this study are generally agreement with seawater bacterial communities in shellfish hatcheries, being dominated by Proteobacteria- (predominantly Gammaproteobacteria and Alphaproteobacteria) and Bacteroidetes -(predominantly Flavobacteria) affiliated sequences. Gammaproteobacteria and/or Alphaproteobacteria have been found as dominant members of the bacterial populations in the water from different Great scallop larvae aquaculture systems [2]. Analyses conducted using Gammaproteobacteria specific probes have also found significant percentages (9\% $48 \%$ ) of this bacterial group in total counts of water samples from Chilean scallop larvae culture tanks [17]. A high fraction of the culturable bacteria (60\%) are assigned to Gammaproteobacteria, as observed by Godoy et al., in the water of Chilean scallop larvae reared [3]. The bacterial communities in the intake and treated clean seawater from a commercial C. gigas hatchery are dominated by the Alphaproteobacteria, Gammaproteobacteria and Flavobacteria [16]. In addition, Gammaproteobacteria, Alphaproteobacteria and CFB group (Bacteroidetes) have also been detected from seawater in a marine hatchery surrounding waters [18]. Of 12 genera (Figure 2) described in this study, Pseudoalteromonas and Vibrio have been detected in the water from Great scallop larvae aquaculture systems [2], Alteromonas is one of the dominant genera in the water of Chilean scallop larval-rearing tanks [3], and Glaciecola and Polaribacter are common in the seawater used for the oyster C. gigas hatchery [16]. Vibrio, Alteromonas,
Pseudoalteromonas, Shewanella and Marinomonas can be isolated from growing-seawater of Ostrea edulis [19]. Sulfitobacter, Phaeobacter, Allivibrio and Cobetia, and some OTU unclassified to genus level, such as NS3a marine group, Roseobacter clade DC5-80-3 lineage and AS-21 lineage have been found from coastal seawater [2024]. Neptuniibacter was first isolated from surface water of the eastern Mediterranean Sea [25].

In this study, relative abundances of Aliivibrio and Vibrio during stages W2 and W3 were higher than those at other 3 stages (Figure 2, Figure 4), which might be related larval mortality occurred during the transition from Dshaped to umbo larvae and at the entire period of stage $\mathrm{W} 4$. Vibrio splendidus V1 and V. tasmaniensis V2 strains have been found to be associated with mass mortality of Yesso scallop larvae [1]. The sequences of OTU $102(\mathrm{~V}$. splendidus) at stages W2 and W3 was more abundant than that at other 3 stages, which might be the source of larval pathogen due to the fact that higher abundance of OTU 102 from larvae was observed at stages $\mathrm{W} 3$ and $\mathrm{W} 4$ [7]. $\mathrm{V}$. splendidus has also been identified as the pathogen for the mass mortalities of Great scallop and Chilean scallop larvae [26-27]. A Vibrio anguillarum-related strain pathogenic to Chilean scallop larvae has been identified [28]. Vibrio pectenicida has been isolated and described by Lambert et al. as being the major cause of mortality during the production of Great scallop larvae in France. Regarding the bacteria in the present study, an assessment of the virulence of species in genus Aliivibrio in the Yesso scallop larvae is warranted [29].

Kesarcodi-Watson et al. has screened probiotics for Greenshell $^{\mathrm{TM}}$ mussel Perna canaliculus larvae from three sources including hatchery seawater [30]. Pseudoalteromonas haloplanktis (formerly Alteromonas haloplanktis) and Vibrio sp. are able to confer protection to Chilean scallop larvae against infection of pathogenic $V$. anguillarum [31-32]. For the larval rearing of Great scallop, protection against Vibrio coralliilyticus and $V$. splendidus is provided by prior administration of Phaeobacter allaeciensis and Alteromonas macleodii 0444. Significant protection with Pseudoalteromonas sp. D41 is only provided against $V$. splendidus [33]. However, whether the bacteria in genera of Pseudoalteromonas, Vibrio, Phaeobacter and Alteromonas found in this study have a probiotic effect in Yesso scallop larvae culture is unclear and requires investigation.

\section{Conclusions}

In summary, water bacterial community compositions were diverse across five Yesso scallop larval development stages, especially in the middle stages. With our 


\section{International Journal of Oceanography \& Aquaculture}

knowledge, this study is the first to use high-throughput sequencing to examine, in detail, the bacterial community in the water of scallop larval-rearing system. It extended current knowledge on microbial community in a scallop hatchery.

\section{Acknowledgements}

We acknowledge the financial support from Zhang Zidao Sland Group Company Limited for the project (99801214).

\section{References}

1. Liu JC, Sun XY, Li M, Zhang CY, Cao SQ, et al. (2015) Vibrio infections associated with Yesso scallop (Patinopecten yessoensis) larval culture. Journal of Shellfish Research 34(2): 213-216.

2. Sandaa R, Magnesen T, Torkildsen L, Bergh L (2003) Characterisation of the bacterial community associated with early stages of great scallop (Pecten maximus), using denaturing gradient gel electrophoresis (DGGE). Syst Appl Microbiol 26(2): 302-311.

3. Godoy FA, Espinoza M, Wittwer G, Uriarte I, Aranda C (2011) Characterization of culturable bacteria in larval cultures of the Chilean scallop Argopecten purpuratus. Ciencias Marinas 37(3): 339-348.

4. Shendure J, Ji H (2008) Next-generation DNA sequencing. Nature Biotechnology 26: 1135-1145.

5. Brown MV, Philip GK, Bunge JA, Smith MC, Bissett A, et al. (2009) Microbial community structure in the North Pacific Ocean. ISME J 3(12): 1374-1386.

6. Dong Y, Zhao Y, Zhang WY, Li Y, Zhou F, et al. (2014) Bacterial diversity and community structure in the East China Sea by 454 sequencing of the 16S rRNA gene. J Ocean Limnol 32(3): 527-541.

7. Sun XY, Liu JC, Li M, Zhao X W, Liang J, et al. (2016) Characterization of bacterial communities associating with larval development of yesso scallop (Patinopecten yessoensis jay, 1857) by highthroughput sequencing. J Ocean Univ China 15(6): 1067-1072.

8. Lane DJ (1991) 16S/23S rRNA sequencing. In: Stackebrandt E, Goodfellow MD (eds) Nucleic Acid Techniques in Bacterial Systematic. 1st (edn) John Wiley and Sons, Chichester, New York, pp: 115-175.
9. McBain AJ, Bartolo RG, Catrenich CE, Charbonneau D, Ledder RG, et al. (2003) Microbial characterization of bio films in domestic drains and the establishment of stable biofilm microcosms. Appl Environ Microbiol 69(1): 177-185.

10. Caporaso JG, Kuczynski J, Stombaugh J, Bittinger K, Bushman FD, et al. (2010) QIIME allows analysis of high-throughput community sequencing data. Nat Methods 7(5): 335-336.

11. Edgar RC, Haas BJ, Clemente JC, Quince C, Knight R (2011) UCHIME improves sensitivity and speed of chimera detection. Bioinformatics 27(16): 21942200.

12. Edgar RC (2013) UPARSE: Highly accurate OTU sequences from microbial amplicon reads. Nat Methods 10: 996-998.

13. Wang Q, Garrity GM, Tiedje JM, Cole JR (2007) Naive Bayesian classifier for rapid assignment of rRNA sequences into the new bacterial taxonomy. Appl Environ Microbiol 73(16): 5261-5267.

14. Quast C, Pruesse E, Yilmaz P, Gerken J, Schweer T, et al. (2013) The SILVA ribosomal RNA gene database project: improved data processing and web-based tools. Nucleic Acids Res 41: 590-596.

15. Amato KR, Yeoman CJ, Kent A, Righini N, Carbonero F, et al. (2013) Habitat degradation impacts black howler monkey (Alouatta pigra) gastrointestinal microbiomes. ISME J 7(7): 1344-5313.

16. Powell SM, Chapman CC, Bermudes M, Tamplin ML (2013) Dynamics of seawater bacterial communities in a shellfish hatchery. Microb Ecol 66: 245-256.

17. Jorquera M, Leyton L, Riquelme C (2004) Bacteria of subclass $\gamma$-proteobacteria associated with commercial Argopecten purpuratus (Lamark 1819) hatcheries in Chile. Aquaculture 236(1-4): 37-51.

18. Schulze AD, Alabi AO, Tattersall-Sheldrake AR, Miller KM (2006) Bacterial diversity in a marine hatchery: Balance between pathogenic and potentially probiotic bacterial strains. Aquaculture 256 (1-4): 50-73.

19. Pujalte MJ, Ortigosa M, Macián MC, Garay E (1999) Aerobic and facultative anaerobic heterotrophic bacteria associated to Mediterranean oysters and seawater. Int Microbiol 2: 259-266.

20. Arahal DR, Castillo AM, Ludwig W, Schleifer KH, 


\section{International Journal of Oceanography \& Aquaculture}

Ventosa A (2002) Proposal of Cobetia marina gen. nov., comb. nov., within the family Halomonadaceae, to include the species Halomonas marina. Syst Appl Microbiol 25(2): 207-211.

21. Buchan A, González JM, Moran MA (2005) Overview of the marine Roseobacter lineage. Appl Environ Microbiol 71: 5665-5677.

22. Kwak MJ, Lee JS, Lee KC, Kim KK, Eom MK, et al. (2014) Sulfitobacter geojensis sp. nov., Sulfitobacter noctilucae sp. nov., and Sulfitobacter noctilucicola sp. nov., isolated from coastal seawater. Int J Syst Evol Microbiol 64(Pt 11): 3760-3767.

23. Bobrova OE, Kristoffersen JB, Ivanytsia VO (2015) Metagenome 16S rRNA gene analysis of the black sea microbial diversity in the region of the Zmiiniy Island. Microbiology and Biotechnology 2: 6-19.

24. Teeling H, Fuchs BM, Bennke CM, Krüger K, Chafee M, et al. (2016) Recurring patterns in bacterioplankton dynamics during coastal spring algae blooms. ELife 5: e11888.

25. Arahal DR, Lekunberri I, González JM, Pascual J, Pujalte MJ, et al. (2007) Neptuniibacter caesariensis gen. nov., sp. nov., a novel marine genome-sequenced gammaproteobacterium. Int J Syst Evol Microbiol 57(Pt 5): 1000-1006.

26. Torkildsen L, Lambert C, Nylund A, Magnesen T, Bergh $\varnothing ~(2005)$ Bacteria associated with early life stages of the Great scallop, Pecten maximus: impact on larval survival. Aquacult Int 13(6): 575-592.

27. Rojas R, Miranda CD, Opazo R, Romero J (2015) Characterization and pathogenicity of Vibrio splendidus strains associated with massive mortalities of commercial hatchery-reared larvae of scallop Argopecten purpuratus (Lamarck, 1819). J Invertebr Pathol 124: 61-69.
28. Riquelme C, Hayashida G, Toranzo AE, Vilches J, Chavez P (1995) Pathogenicity studies of a Vibrio anguillarum related (VAR) strain causing an epizootic in Argopecten purpuratus larvae cultured in Chile. Inter-Research 22: 135-141.

29. Lambert C, Nicolas JL, Cilia V, Corre S (1998) Vibrio pectenicida sp. nov., a pathogen of scallop (Pecten maximus) larvae. Int J Syst Bacteriol 48(Pt 2): 481487.

30. Kesarcodi-Watson A, Kaspar H, Lategan MJ, Gibson, L (2009) Screening for probiotics of Greenshell ${ }^{\text {TM }}$ mussel larvae, Perna canaliculus, using a larval challenge bioassay. Aquaculture 296(1-2): 159-164.

31. Riquelme C, Hayashida G, Araya R, Uchida A, Satomi $M$, et al. (1996) Isolation of a native bacterial strain from the scallop Argopecten purpuratus with inhibitory effects against pathogenic vibrios. Journal of Shellfish Research 15(2): 369-374.

32. Riquelme C, Araya R, Vergara N, Rojas A, Guaita M, et al. (1997) Potential probiotic strains in the culture of the Chilean scallop Argopecten purpuratus (Lamarck, 1819). Aquaculture 154: 17-26.

33. Kesarcodi-Watson A, Miner P, Nicolas JL, Robert, R (2012) Protective effect of four potential probiotics against pathogen-challenge of the larvae of three bivalves: Pacific oyster (Crassostrea gigas), flat oyster (Ostrea edulis) and scallop (Pecten maximus). Aquaculture 344-349: 29-34. 